\title{
Les métabolites de la vitamine $D$ : leur action sur l'os des rats carencés en vitamine D
}

\author{
par Livia MIRAVET $\left({ }^{(}\right)$, M. L. QUEILLE, M. CARRE, Ph. BORDIER, J. REDEL * \\ Unifé de Recherche sur le Méfabolisme Phosphocalcique \\ U. 18 INSERM, Hôpital Lariboisière, 6-8 rue Guy-Patin, 75010 Paris. \\ * Institut de Rhumatologie, Centre de Recherche sur les Maladies \\ Ostéo-articulaires \\ U. 5 INSERM-ERA 337 CNRS, Hôpital Cochin 75006 Paris.
}

Summory. Action of vitamin D metabolites on the bone of vitamin D-deficient rats.

The effect of $25 \mathrm{OHD}_{3}, 1.25(\mathrm{OH})_{2} \mathrm{D}_{3}, 24,25(\mathrm{OH})_{2} \mathrm{D}_{3}$ and $25,26(\mathrm{OH})_{2} \mathrm{D}_{3}$ was studied on the bone of vitamin D-deficient rats. The products were administered by intraperitoneal route during 5 days at a dose of $325 \mathrm{pmol} / \mathrm{day}$. The animals were killed 24 hours after the last injection. Serum calcium was significantly increased by all metabolites, but the increment was not similar in all cases; serum phosphorus was only increased by $25 \mathrm{OHD}_{3}$ and $1.25(\mathrm{OH})_{2} \mathrm{D}_{3}$. Bone calcium content increased after all treatments, but the bone phosphorus content augmented only after $25,26(\mathrm{OH})_{2} \mathrm{D}_{3}$ treatment. Histological studies showed that $25 \mathrm{OHD}_{3}$ had an osteoblastic action and $1.25(\mathrm{OH})_{2} \mathrm{D}_{3}$ an osteoclastic action. $24,25(\mathrm{OH})_{2} \mathrm{D}_{3}$ calcified the osteoid and increased the number of osteoclasts, but not their activity. $25,26(\mathrm{OH})_{2} \mathrm{D}_{3}$ simulated calcification without any increment of osteoclastic activity. The bone of $25,26(\mathrm{OH})_{2} \mathrm{D}_{3}$-treated rats was similar to the bone of normal animals.

Quatre dérivés vitaminiques $\mathrm{D}$ ont été décelés dans le plasma : le 25 hydroxycholécalciférol $\left(25 \mathrm{OH} \mathrm{D}_{3}\right)$, le 1,25 dihydroxycholécalciférol $\left(1,25(\mathrm{OH})_{2} \mathrm{D}_{3}\right)$, le 24.25 dihydroxycholécalciférol $\left(24,25(\mathrm{OH})_{2} \mathrm{D}_{3}\right)$ et le 25,26 dihydroxycholécalciférol $(25,26$ $(\mathrm{OH})_{2} \mathrm{D}_{3}$ ) (De Luca, 1976). L'action biologique de la vitamine s'effectue par l'intermédiaire de ces dérivés hydroxylés.

L'action intestinale sur l'absorption calcique est propre à la vitamine $D$, en son absence l'absorption calcique esi toujours très déficiente. L'hydroxylation en $\mathrm{C} 1$ a été reconnue essentielle pour induire l'absorption calcique intestinale : le $1,25(\mathrm{OH})_{2} \mathrm{D}_{3}$ est le plus actif des dérivés vitaminiques connus pour cette action, le $24,25(\mathrm{OH})_{2} \mathrm{D}_{3}$ et le $25,26(\mathrm{OH})_{2} \mathrm{D}_{3}$ doivent s'hydroxyler en $\mathrm{C} 1$ pour être actifs sur l'absorption calcique intestinale puisque la néphrectomie totale fait disparaître leur action (De Luca 1976 ; Miravet ef al., 1976).

(1) Tirés à Part : Miravet Livia, 6-8 rue Guy-Patin, 75010 Paris, France. 
L'activité antirachitique ou calcifiante est encore considérée par certains comme une action indirecte résultant de la normalisation humorale du calcium et du phosphore, grâce à l'action produite par la vitamine D sur l'absorption calcique ef la possibilité de mobiliser le calcium osseux, les deux tendant à élever le calcium et le phosphore plasmatique.

L'action osseuse des trois dihydroxylés vitaminiques n'est pas encore bien connue; nous avons voulu éfudier cette action en administrant séparément chacun d'entre eux à des animaux carencés en vitamine $D$.

\section{Matériel et méthodes.}

Des rats Sprague-Dawley (Charles River-France) ont été mis au sevrage dans des cages individuelles dans une pièce noire et nourris pendant trois semaines avec une diète semi-synthétique (La Minière-INRA) dépourvue de vitamine $D$. D'après les expériences l'apport calcique et phosphoré a été normal $(0,47$ p. 100 et 0,30 p. 100) ou pauvre en calcium $(<0,02$ p. 100) ou pauvre en phosphore $(<0,02$ p. 100), l'autre élément restant normal.

Les produits utilisés ont été : le $25 \mathrm{OH} \mathrm{D}_{3}$ (Roussel), le $1,25(\mathrm{OH})_{2} \mathrm{D}_{3}$ produił dans notre laboratoire par biosynthèse (Carré ef al., 1974) ; le $24,25(\mathrm{OH})_{2} \mathrm{D}_{3}$ et le $25,26(\mathrm{OH})_{2} D_{3}$ ont été synthétisés par l'un d'entre nous en partant du 3 bêtahydroxy-25nor-5-cholesten-25-one (Redel ef al., 1974, 1975). Les produits obtenus sont un mélange de deux diastéro-isomères $R$ et $S$. Les administrations ont été faites par voie intraveineuse pour les expériences avec une injection et par voie intra-péritonéale quand il y eu injections multiples.

Le sang a été prélevé par ponction cardiaque ; dans le plasma nous avons dosé le calcium par absorption atomique dans une solution spectrale à 5 p. 1000 de $\mathrm{LaCl}$ (Perkin Elmer 303) et le phosphore par le Kit Biotrol.

Au moment du sacrifice, nous avons prélevé les tibias et fémurs. Après nettoyage des parties molles adhérentes et de la moelle, les os ont été séchés et calcinés à $600^{\circ} \mathrm{C}$, les cendres ont été reprises dans $\mathrm{HCl} \mathrm{N}$ ef le calcium et le phosphore ont été dosés. Les vertèbres caudales ( $9-10$ et 11) ont été fixées dans le formol à 10 p. 100, incluses sans décalcification dans du métacrylate et coupées à $5 \mu \mathrm{m}$ dans un microtome Yung ; les comptages semi-quantitatifs ont éfé faits d'après la technique décrite précédemment (Bordier et Tun-Chot, 1972).

L'absorption calcique a été mesurée in vitro d'après la technique de Schachter et Rosen (1959).

La mobilisation calcique osseuse a été mesurée d'après les variations de la calcémie chez des animaux ingérant une dièłe très pauvre en calcium.

\section{Résultats.}

1. Action des différents dérivés sur l'absorption calcique intestinale.

Le 1,25 (OH $)_{2} D_{3}$ est le seul dérivé capable d'améliorer l'absorption calcique intestinale dans n'importe quelle situation, même après néphrectomie (Boyle ef al., 1972). 
Le $24,25(\mathrm{OH})_{2} \mathrm{D}_{3}$ ef le $25,26(\mathrm{OH})_{2} \mathrm{D}_{3}$ (fig. 1) n'augmentent significativement l'absorption calcique intestinale que chez les animaux soumis à une diète pauvre en calcium, situation dans laquelle l'hydroxylation en C1 est maximale.

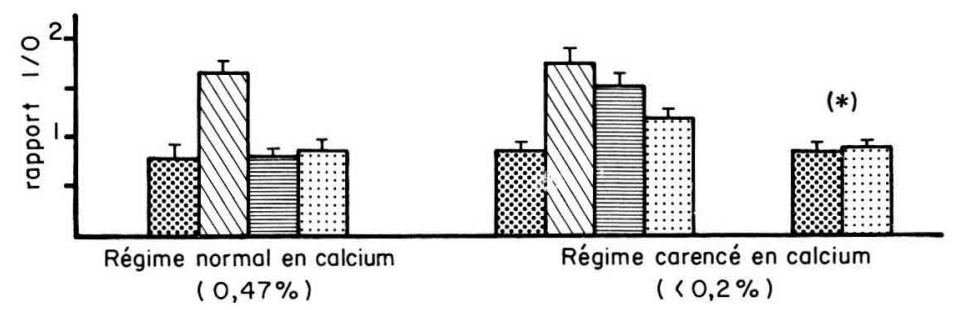

(*) animaux binephrectomisés

$\begin{array}{llll}93 & \text { corencés } & 24.25 & (\mathrm{OH})_{2} \mathrm{CC} \\ 25 \mathrm{OHCC} & 25.26 & (\mathrm{OH})_{2} \mathrm{CC}\end{array}$

FIG. 1. - Action des différents métabolites de la Vitamine $D_{3}$ sur l'absorption calcique intestinale mesurée in vitro, selon la technique de l'anse duodénale éversée.

\section{TABLEAU 1}

Dosages plasmatiques du calcium ef phosphore $24 \mathrm{~h}$ après une injection intraveineuse chez des rats déficients en vitamine $D$

Régime normal en calcium ef phosphore

\begin{tabular}{|c|c|c|c|c|}
\hline Produit injecté & $\begin{array}{l}\text { Dose } \\
\text { (pmol) }\end{array}$ & $\begin{array}{c}\text { Nombre } \\
\text { d'animaux }\end{array}$ & $\begin{array}{l}\text { Calcémie } \\
(\mathrm{mg} / \mathrm{l})\end{array}$ & $\begin{array}{c}\text { Phosphorémie } \\
(\mathrm{mg} / \mathrm{l})\end{array}$ \\
\hline $\begin{array}{l}\text { Alcool } \ldots \ldots \ldots \ldots \ldots \ldots \ldots \\
25 \text { OHD } \mathrm{H}_{3} \ldots \ldots \ldots \ldots \ldots \ldots \\
1,25(\mathrm{OH})_{2} \mathrm{D}_{3} \ldots \ldots \ldots \ldots \ldots \ldots \\
24,25(\mathrm{OH})_{2} \mathrm{D}_{3} \ldots \ldots \ldots \ldots \ldots \\
25,26(\mathrm{OH})_{2} \mathrm{D}_{3} \ldots \ldots \ldots \ldots \ldots\end{array}$ & $\begin{array}{l}50 \mu l \\
650 \\
325 \\
325 \\
650 \\
650\end{array}$ & $\begin{array}{r}6 \\
6 \\
5 \\
5 \\
10 \\
10\end{array}$ & $\begin{array}{l}48 \pm 6 \\
77 \pm 5 \\
85 \pm 5 \\
63 \pm 13 \\
54 \pm 8 \\
51 \pm 7\end{array}$ & $\begin{array}{l}64 \pm 14 \\
68 \pm 4 \\
49 \pm 11 \\
50 \pm 15 \\
68 \pm 7 \\
59 \pm 6\end{array}$ \\
\hline
\end{tabular}

Régime pauvre en calcium

\begin{tabular}{|c|c|c|c|c|}
\hline $\begin{array}{l}\text { Alcool } \ldots \ldots \ldots \ldots \ldots \ldots \\
25 \text { OHD } \mathrm{D}_{3} \ldots \ldots \ldots \ldots \ldots \ldots \ldots \\
1,25(\mathrm{OH})_{2} \mathrm{D}_{3} \ldots \ldots \ldots \ldots \ldots \ldots \\
24,25(\mathrm{OH})_{2} \mathrm{D}_{3} \ldots \ldots \ldots \ldots \ldots \ldots \\
25,26(\mathrm{OH})_{2} \mathrm{D}_{3} \ldots \ldots \ldots \ldots \ldots \ldots\end{array}$ & $\begin{array}{l}50 \mu l \\
650 \\
650 \\
650 \\
650\end{array}$ & $\begin{array}{l}5 \\
5 \\
8 \\
5 \\
5\end{array}$ & $\begin{array}{l}47 \pm \\
72 \pm \\
73 \pm \\
48 \pm 13 \\
45 \pm\end{array}$ & $\begin{array}{l}65 \pm \\
93 \pm 1 \\
66 \pm \\
62 \pm\end{array}$ \\
\hline
\end{tabular}

Régime pauvre en phosphore

\begin{tabular}{|c|c|c|c|c|}
\hline $\begin{array}{l}\text { Alcool } \ldots \ldots \ldots \\
1.25(\mathrm{OH})_{2} \mathrm{D}_{3} \ldots \ldots \ldots \ldots \ldots \ldots \\
24.25(\mathrm{OH})_{2} \mathrm{D}_{3} \ldots \ldots \ldots \ldots \ldots \\
25.26(\mathrm{OH})_{2} \mathrm{D}_{3} \ldots \ldots \ldots \ldots \ldots \ldots\end{array}$ & $\begin{array}{l}50 \mu l \\
325 \\
325 \\
650 \\
650\end{array}$ & $\begin{array}{r}4 \\
4 \\
5 \\
13 \\
14\end{array}$ & $\begin{array}{r}106 \pm 6 \\
109 \pm 13 \\
79 \pm 15 \\
79 \pm 9 \\
80 \pm 8\end{array}$ & $\begin{array}{l}22 \pm 4 \\
33 \pm 9 \\
35 \pm 4 \\
29 \pm 10 \\
25 \pm 10\end{array}$ \\
\hline
\end{tabular}


La néphrectomie totale préalable fait disparaître la possibilité d'améliorer l'absorption calcique intestinale, autant pour le $24,25(\mathrm{OH})_{2} \mathrm{D}_{3}(\mathrm{De} \mathrm{Luca}, 1976)$ que pour le $25.26(\mathrm{OH})_{2} \mathrm{D}_{3}$ (Miravet et al., 1976).

Action aiguë des différents dérivés sur la calcémie (łabl. 1). La calcémie des animaux témoins varie d'après le régime minéral qu'ils ont reçu préalablement.

Quant les animaux sont maintenus dans un régime normal ou pauvre en calcium, la calcémie est très diminuée au départ, le calcium plasmatique augmente significativement 24 heures après l'administration intra-veineuse de $25 \mathrm{OHD}_{3}$ ef le $1,25(\mathrm{OH})_{2} \mathrm{D}_{3}$ Dans ces conditions, le $24,25(\mathrm{OH})_{2} \mathrm{D}_{3}$ et le $25,26(\mathrm{OH})_{2} \mathrm{D}_{3}$ ne sont pas capables de produire une élévation de la calcémie importante.

Lorsque les animaux onf eu un régime pauvre en vitamine $D$ ef phosphore, leur calcémie est normale, le $1,25(\mathrm{OH})_{2} D_{3}$ ne l'élève que très peu et le $24,25(\mathrm{OH})_{2} \mathrm{D}_{3}$ ef le $25,26(\mathrm{OH})_{2} \mathrm{D}_{3}$ la font diminuer de façon significative.

3. Action aiguë des différents dérivés sur le phosphore plasmatique. La phosphorémie (tabl. 1) est pratiquement normale chez les animaux en régime normal ou pauvre en calcium et augmente de manière significative $24 \mathrm{~h}$ après administration intra-veineuse de $25 \mathrm{OH} \mathrm{D}_{3}$, en régime pauvre en calcium. Malheureusement, dans cefte expérience, le phosphore des animaux qui avaient reçu du 1,25(OH) $)_{2} D_{3}$ n'a pas été dosé.

Dans aucune condition alimentaire, le phosphore n'augmente après administration du 24,25 (OH $)_{2} \mathrm{D}_{3}$ ou du 25,26(OH) $)_{2} \mathrm{D}_{3}$.

4. Modifications du calcium ef du phosphore plasmatique après administrations répéfées des dérivés vitaminiques. Si au lieu d'administrer une dose unique de dérivés vitaminiques par voie intra-veineuse, on injecte par voie péritonéale cinq jours consécutifs 325 pmol, les résultats ne sont pas identiques aux précédents (fig. 2).

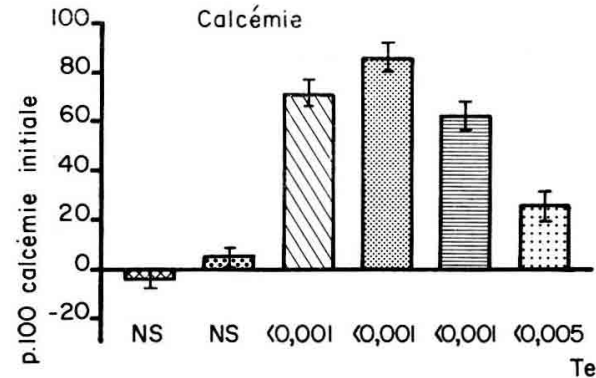

Test de Student

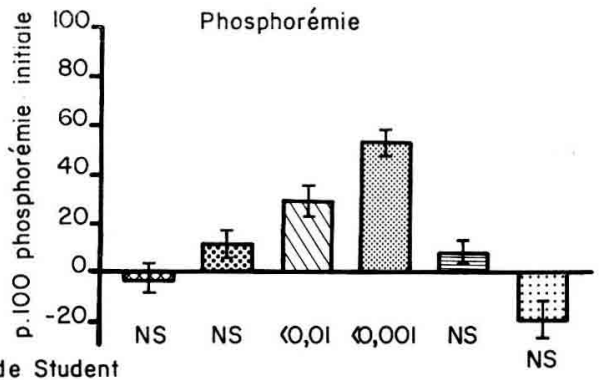

$24-25(\mathrm{OH})_{2} \mathrm{CC}$ $25 \_26(\mathrm{OH})_{2} \mathrm{CC}$

FIG. 2. - p. 100 d'augmentation de la calcémie ou de la phosphorémie, par rapport aux taux témoins du même groupe des rots traités par voie intra-péritonéale pendant cinq jours avec 325 pmol de chaque métabolife. Les rats considérés comme normaux ont reçu, depuis le sevrage jusqu'au sacrifice (4 semaines), $195 \mathrm{pmol} / \mathrm{j}$ de vitamine $\mathrm{D}_{3}$. Les rats carencés contrôlés ont reçu une injection intrapéritonéale de $50 \mu \mathrm{l}$ d'alcool pendant cinq jours.

La calcémie augmente avec tous les dérivés : le $25 \mathrm{OH} \mathrm{D}_{3}$ la ramène à la normale $(106 \pm 0,6 \mathrm{mg} / 1)$, le $1,25(\mathrm{OH})_{2} D_{6}$ l'élève légèrement au-dessus de celle des animaux témoins $(114 \pm 3 \mathrm{mg} / \mathrm{l})$, le $24,25(\mathrm{OH})_{2} \mathrm{D}_{3}(84,2 \pm 3 \mathrm{mg} / \mathrm{l})$ ef le $25,26(\mathrm{OH})_{2} \mathrm{D}_{3}$ 
$(82 \pm 4 \mathrm{mg} / \mathrm{l})$ l'augmentent de manière significative mais elle reste très en dessous de celle des animaux normaux $(102,5 \pm 2,5 \mathrm{mg} / \mathrm{l})$.

Le phosphore plasmatique est franchement augmenté par le $25 \mathrm{OH} \mathrm{D}_{3}(90 \pm$ $2,6 \mathrm{mg} / 1)$, ainsi que par le 1,25 $(\mathrm{OH})_{2} \mathrm{D}_{3}(76 \pm 3,3 \mathrm{mg} / \mathrm{l})$, par contre les phosphorémies des animaux ayant reçu le $24,25(\mathrm{OH})_{2} \mathrm{D}_{3}(66 \pm 3 \mathrm{mg} / \mathrm{l})$ et le $25,26(\mathrm{OH})_{2} \mathrm{D}_{3}$ $(69 \pm 5 \mathrm{mg} / \mathrm{l})$ sont identiques à celles des animaux normaux $(63 \pm 5 \mathrm{mg} / \mathrm{l})$.

5. Calcium et phosphore des cendres osseuses (fig. 3). Ils ont été mesurés après le traitement de cinq jours par voie intrapéritonéale.

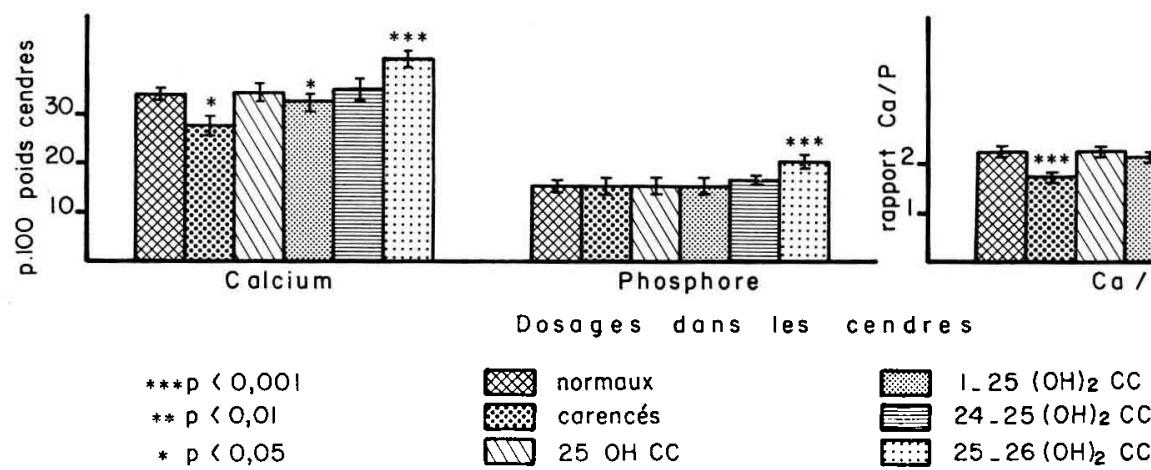

FIG. 3. - Calcium et phosphore dans les cendres.

Le calcium des cendres, qui était significativement diminué chez les carencés par rapport aux normaux, augmente significativement avec les quatre dérivés vitaminiques utilisés. Mais, contrairement à leurs effets sur la calcémie, le moins efficace est le $1,25(\mathrm{OH})_{2} \mathrm{D}_{3}$ et le plus efficace le $25.26(\mathrm{OH})_{2} \mathrm{D}_{3}$.

La teneur en phosphore est identique chez l'animal normal et chez l'animal carencé en vitamine $D$; ni le $25 \mathrm{OH} \mathrm{D}_{3}$, ni le $1,25(\mathrm{OH})_{2} \mathrm{D}_{3}$, ni le $24,25(\mathrm{OH})_{2} \mathrm{D}_{3}$ ne font changer le contenu en phosphore osseux. Le seul dérivé qui semble capable d'améliorer la rétention du phosphore dans l'os, est le $25,26(\mathrm{OH})_{2} \mathrm{D}_{3}$.

6. Etude histologique osseuse. Nous avons déjà constaté (Gruson et al., 1974), que le $1,25(\mathrm{OH})_{2} \mathrm{D}_{3}$ a essentiellement un pouvoir ostéolytique osseux par rapport à l'effet observé avec la même dose de $25 \mathrm{OH} \mathrm{D}_{3}$.

Les résultats des comptages semi-quantitatifs, qui ont été publiés en détail (Queillé et al., 1977), nous ont montré que le $25 \mathrm{OH} \mathrm{D}_{3}$ stimule l'ostéoblastose et freine l'ostéolyse en diminuant à la fois le nombre d'ostéoclastes et les surfaces de résorption. Le $1,25(\mathrm{OH})_{2} \mathrm{D}_{3}$ a un pouvoir calcifiant intense, mais les surfaces d'activité ostéoblastiques sont réduites ; par ailleurs, il élève considérablement le nombre d'ostéoclastes ef active leur fonctionnement, car les surfaces de résorption sont très augmentées par rapport aux rats normaux.

Le 24, $25(\mathrm{OH})_{2} D_{3}$ a un effet très particulier : il augmente les fronts de calcification, mais le volume ostéoïde restant est peu différent de celui des animaux carencés en vitamine $D$. Sur la résorption, son activité est également dissociée, car le nombre d'ostéoclastes augmente mais les surfaces de résorption active ne changent pas.

Le $25,26(\mathrm{OH})_{2} D_{3}$ stimule la calcification (les fronts de calcification augmentent), 
diminue le volume ostéoïde des animaux carencés, sans pour autant le ramener à celui des animaux normaux, mais ne modifie guère les facteurs de résorption : ostéoclastes ou surfaces de résorption. Parmi les dérivés utilisés, le $25,26(\mathrm{OH})_{2} \mathrm{D}_{3}$ est celui qui rapproche le plus l'os des animaux rachitiques de celui des animaux normaux.

\section{Discussion.}

Après avoir identifié les dérivés vitaminiques dihydroxylés nous avions considéré le $1,25(\mathrm{OH})_{2} \mathrm{D}_{3}$ comme étant le seul dérivé actif, les autres $24,25(\mathrm{OH})_{2} \mathrm{D}_{3}$ et 25 , $26(\mathrm{OH})_{2} \mathrm{D}_{3}$ étant alors des produits du catabolisme ou d'élimination.

Depuis, quelques faits troublants furent observés qui mirent en doute l'importance capitale du $1,25(\mathrm{OH})_{2} D_{3}$ pour la calcification, bien qu'il soit le dérivé le plus actif pour normaliser la calcémie des animaux ou de l'homme en état de carence, et qu'il soif également le plus actif, tout au moins chez le rat, sur l'élévation de la phosphatémie abaissée (Garabedian ef al., 1976), la possibilité de calcification n'a été mise en évidence qu'avec difficulté (Tanaka ef al., 1972). Chez l'homme néphrectomisé total (Bordier et al., 1973), l'ostéomalacie n'est pas toujours présente, bien que toute possibilité d'hydroxylation en $\mathrm{Cl}$ soit perdue. De même, chez l'ostéomalacique, s'il est vrai que le $1,25(\mathrm{OH})_{2} D_{3}$ occasionne un début de guérison (Bordier et al., 1970, 1977), même des traitements prolongés ne sont pas capables de rendre totale.

De plus, lors de l'équilibre vitaminique la quantité circulante de 1,25 $(\mathrm{OH})_{2} \mathrm{D}_{3}$ est infime $(0,02$ à $0,03 \mathrm{ng} / \mathrm{ml}$, Haussler et al., 1975), alors que d'autres dérivés, et en particulier le 24, $25(\mathrm{OH})_{2} \mathrm{D}_{3}$ sont bien plus importants (2 à $3 \mathrm{ng} / \mathrm{ml}$; Garabedian ; Comm. personnelle). Le troisième dihydroxylé le $25,26(\mathrm{OH})_{2} \mathrm{D}_{3}$ existerait en quantité non négligeable (Mawer et al., 1974), mais les techniques utilisées jusqu'à présent n'ont pas permis de le séparer convenablement du $1,25(\mathrm{OH})_{2} \mathrm{D}_{3}$.

Lorsque nous avons commencé cette étude, les doses circulantes des différents dérivés dihydroxylés n'étaient pas encore connues ; nous avons donc employé des doses comparables pour tous les métabolites et il est probable que l'équivalence molaire nous a conduit à un hyperdosage pour le $1,25(\mathrm{OH})_{2} D_{3}$. Néanmoins, les résultats obtenus, tant sur les constantes biologiques lors des expériences aiguës, que sur l'os pendant l'expérience semi-chronique, nous permettent de suspecter la nécessité de tous ces métabolites pour l'équilibre vitaminique osseux.

Si pour l'absorption intestinale calcique il est certain que la présence de l'hydroxylé en $\mathrm{C} 1$ esł indispensable, et que le rein semble encore être le seul organe capable de réaliser cette hydroxylation, l'action osseuse est vraisemblablement plus complexe.

De l'étude que nous avons réalisée, nous pouvons au moins conclure que l'action sur la calcification est en effet vitaminique direct et non proportionnel aux variations de la calcémie, puisque les dihydroxylés qui permettent une plus importante rétention du calcium dans l'os, $24,25(\mathrm{OH})_{2} \mathrm{D}_{3}$ et le $25,26(\mathrm{OH})_{2} \mathrm{D}_{8}$, sont ceux qui augmentent le moins la calcémie. De plus, le seul qui retienne le phosphore $\left(25,26(\mathrm{OH})_{2} \mathrm{D}_{3}\right)$, au lieu de faire augmenter la phosphorémie, la fait diminuer de manière cependant non significative.

Même si les effets observés ne sont pas produits directement par la substance injectée, étant donné l'expérience « in vivo» qui a permis peut-être une métabolisation postérieure, il ne reste pas moins vrai que la réponse est loin d'être identique et que dans aucun cas, elle n'est nulle, ce qui amène à penser que la 
présence de l'hydroxyle supplémentaire dans la chaîne latérale $\left(C_{24}\right.$ ou $\left.C_{26}\right)$, au lieu de faire disparaître l'activité vitaminique sur l'os, la module.

En conclusion, nous pouvons prévoir que l'équilibre d'activité osseuse nécessite la présence des trois dihydroxylés, ef que, très probablement chacun d'entre eux a une fonction particulière à remplir.

Réunion Groupe Développement INRA/Productions onimales Montpellier, 17-18 mai 1977.

\section{Références}

BORDIER Ph., TUN-CHOT S., 1972. Quantitative histology of metabolic bone disease. J. clin. Endocrinol. Med., 1, 197-215.

BORDIER Ph., TUN-CHOT S., EASTWOOD J. B., FOURNIER A., DE WARDENER H. E., 1973. Lack of histological evidence of vitamin $D$ abnormality in the bones of anephric patients. Clin. Sci., 44, 33-41.

BORDIER Ph., TUN-CHOT S., MARTIN J., QUEILLÉ M. L., HIOCO D., 1970. Minéralisation du tissu ostéoïde chez l'ostéomalacique induite par la surcharge phosphorée ou par la vitamine $D_{3}$. Symp. int. Phosphate et Metab. Calcique, Sandoz, Paris.

BORDIER P., RYCKEWAERT A., MARIE P., MIRAVET L., NORMAN A. W., RASMUSSEN H., 1977. Vitamin $D$ metabolites in bone mineralization in man, 897-911. In NORMAN A. W., SCHAEFER K., GRIGOLEIT H. G., HERRATH D. V., RITZ E., Vitamin D and problems related to uremic bone disease. W. de Gruyter, Berlin, NY.

BOYLE I. T., MIRAVET L., GRAY R. W., HOLICK M. F., DE LUCA H. F., 1972. The response of intestinal calcium transport to 25 hydroxy and 1.25 dihydroxy vitamin $D$ in nephrectomized rats. Endocrinology 90, 605-608.

CARRE M., AYGBEDE O., MIRAVET L., RASMUSSEN H., 1974. The effect of prednisolone upon the metabolism and action of 25 hydroxy and 1.25 dihydroxy vitamin $D_{3}$. Proc. nat. Acad. Sci. U.S.A., 71, 2996-3000.

DE LUCA H. F., 1976. Metabolism of vitamin D : current status. In BRONNER F., Symp. Recent Advances in vitamin D : clinical implications. Am. J. clin. Nut., 29, Suppl., 1257-1270.

GARABEDIAN M., PEZANT E., MIRAVET L., FELLOT C., BALSAN S., 1976. 1.25 dihydroxycholecalciferol effect or serum phosphorus homeostasis in rats. Endocrinology, 98, 792-799.

GRUSON M., BORDIER Ph., QUEILLÉ M. L., CARRE M., DEMIGNON J. M., MIRAVET L., 1974. Effects of 25 hydroxycholecalciferol and 1.25 dihydroxycholecalciferol on bone resorption formation and mineralization in vitamin D deficient rats IRCS (Med. Sc), 2, 1154.

HAUSSLER M., 1975. Vitamin $D_{3}$ : metabolism, mode of action and assay of circulating hormonal form, 25-42. In NORMAN A. W., SCHAEFER K., GRIGOLEIT H. G., HERRATH D. V., RITZ E., Vitamin D and problems related to uremic bone disease. W. de Gruyter, Berlin, NY.

MAWER E. B., 1974. Vitamin D metabolism in man. In the metabolism and function of vitamin D Biochem. Soc. Spec. Publ., 3, 27-42.

MIRAVET L., REDEL J., CARRE M., QUEILLÉ M. L., BORDIER Ph., 1976. The biological activity of synthetic 25, 26 dihydroxycholecalciferol and 24, 25 dihydroxycholecalciferol in vitamin $D$ deficient rats. Calcif. Tiss. Res., 21, 145-152.

QUEILLÉ M. L., MIRAVET L., BORDIER Ph., REDEL J., 1977. Action des métabolites de la vitamine D $\left(25 \mathrm{OH} \mathrm{D} \mathrm{D}_{3}-1.25(\mathrm{OH})_{2}-\mathrm{D}_{3}-24,25(\mathrm{OH})_{2}-\mathrm{D}_{3} \cdot 25,26(\mathrm{OH})_{2}-\mathrm{D}_{3}\right.$ sur l'os de rat carencé en vitamine $D$ (soumis à publication).

REDEL J., BAZELY P. A., CALANDO Y., DELBARRE F., BELL P. A., KODICEK E., 1975. The synthesis of 24,25 dihydroxycholecalciferol, a metabolite of vitamin $D_{3}$ J. Steroid Bioch., 6, 117-119.

REDEL J., BELL P. A., BAZELY N., CALANDO Y., DELBARRE F., KODICEK E., 1974. The synthesis and biological activity of 25,26 dihydroxycholecalciferol, a polar metabolite of vitamin $D_{3}$. Steroids, 24, 463-475 (1974).

SCHACHTER D., ROSEN S. M., 1959. Active transport of $45 \mathrm{Ca}$ by the small intestine and its dependance on vitamin D. Amer. J. Physiol., 196, 357-362.

TANAKA Y., FRANK H., DE LUCA H. F., 1972. Role of 1.25 dihydroxycholecalciferol in calcification of bone and maintenance of serum calcium concentration in the rats nutrition. Nutrition, 102, $1568-1578$. 